\title{
Pediatric Neurosurgery
}

Abbassioun, K. 396

Adams, H. 475

Aguilera, D. 303

Ahmed, S. 242

Aihara, Y. 12

Aldana, P.R. 227, 329

Alexiou, G.A. 188, 480

Alho, E.J.L. 249

Al-Holou, W.N. 172

Ali, M.Z. 29

Amiri, R.S. 290

Amirjamshidi, A. 396

Anik, I. 344

Anik, Y. 344

Appelgren, T. 221

Arnold, P.M. 66

Aronin, P.A. 415

Astrand, R. 101

Bahl, A. 462

Balak, N. 110

Balasubramaniam, S. 232

Bansal, A. 46

Barry, S. 409

Bawa, M. 138

Behzadi, A.H. 357

Ben-Haim, S. 193

Bergenheim, A.T. 259

Bhansali, A. 46

Bhat, D.I. 238

Blaivas, M. 326

Boaz, J.C. 457

Bonnin, J.M. 457

Borghei-Razavi, H. 357

Bradshaw, T.J. 141

Braxton, E.E., Jr. 127

Brayton, A. 213

Breithaupt, A. 193

Brezner, A. 82

Browd, S.R. 450

Bukte, Y. 54

Bulbul, A. 199

Bulbul, L.G. 199

Burgan, O.T. 462

Burger, P. 475

Buzunov, E. 120

Campos, W.K. 385

Can, E. 199

Ceylan, S. 344

Charalambides, C. 62

Chavantes, M.C. 249

Chelliah, D. 381
Chen, J. 259

Choi, J.-U. 362

Choulakian, A. 373

Chung, J. 89

Cohen, S.R. 193

Cohen-Gadol, A.A. 71

Cömert, S. 199

Constantini, S. 158

Crabtree, K.L. 66

Critcher, V. 462

Cummings, T.J. 58, 155

Curry, D.J. 141

Dange, N. 39

Danielpour, M. 373, 466

Dauser, R.C. 141

De Biasi, S. 435

Demir, İ. 6, 351

Devi, B.I. 238, 247

Douglas-Akinwande, A.C. 457

Doussis-Anagnostopoulou, I. 188

Drazin, D. 466

Dutta, P. 46

Ehrlich, G.D. 127

Eissa, E.M. 272

Ekici, A. 133

Ekici, M.A. 133

Elfversson, J. 221

El-Gaidi, M.A. 272

Elghandour, M. 427

El Khashab, M. 290

Elmac1, I. 110

El-Shafei, H.I. 160

El-Shafei, I.L. 160

Etus, V. 344

Fangusaro, J. 303

Filardi, T. 450

Fiore, M. 255

Fong, D. 417

Fonseca, A. 39

Fraser, D.D. 318

Fraser, J.F. 151

Frim, D.M. 81, 157, 177

Fuchs, H.E. 58, 155

Fukuda, K. 313

Fulkerson, D.H. 213

Galarza, M. 442

Gallo, P. 435

Gardetto, J. 381
Garg, K. 242

Garton, H.J.L. 172

George, T.M. 415

Ghodke, R. 450

Gilbert, J.W. 408

Giussani, C. 450

Goel, A. 39, 232

Gokben, B. 110

Goldman, S. 303

Grant, G.A. 58, 155

Greenfield, J.P. 151

Gruber, T.J. 19

Güdü, B.O. 351

Guillaume, D.J. 390

Gupta, A. 324

Gupta, C. 368

Gupta, D.K. 242, 267, 472

Gupta, K. 46

Gupta, S.K. 76

Guzel, A. 54

Guzel, E. 54

Hall-Stoodley, L. 127

Ham, S. 255, 442

Han, S.B. 43

Hasanefendioglu Bayrak, A. 54

Hassan, T. 427

Hattab, E.M. 457

Heckmann, M. 34

Heidemann, S.M. 255

Hervey-Jumber, S.L. 326

Hesselgard, K. 101

Higashimoto, Y. 313

Holleman, J. 177

Honda, N. 313

Hori, T. 12

Hughes, B.D. 58

Humphrey, O. 259

Hur, Y.J. 182

Husain, M. 51

Hwang, S.K. 299

Hwang, T. 182

Indelicato, D.J. 227

Isik, N. 110

James, H.E. 227, 329

Javeed, Z. 406

Jea, A. 141, 213

Jhawar, S. 232

Jiang, B. 475

Jödicke, A. 294

Johnson, A. 177
Johnson, K.A. 457

Johnson, S. 127

Jouibari, M.F. 290

Jumper, M. 326

Jung, S. 205

Jung, T.-Y. 205

Kang, J.-A. 43

Kang, S.-S. 205

Kanojia, R.P. 138

Karentzou, O. 188

Karikari, I.O. 155

Kasliwal, M.K. 151, 324

Kathju, S. 127

Kato, A. 313

Kawamata, T. 12

Keshavarzi, S. 193

Keskin, S. 6

Khalatbari, M. 357

Khursheed, N. 406

Kim, D.L. 71

Kim, D.S. 182,362

Kim, H.D. 182

Kim, I.-Y. 205

Kim, J.H. 43

Kim, J.-H. 205

Kim, J.S. 43

Kim, T.-G. 362

Kirmani, A.R. 25

Kiymaz, N. 6, 351

Ko, A. 450

Kostopanagiotou, G. 62

Kovoor, J. 247

Kozan, A. 351

Krebs, V.L.J. 249

Kuchelmeister, K. 294

Kulkarni, A.V. 213

Kurtsoy, A. 133

Kurwale, N.S. 267

Lee, J.A. 146

Lee, J.S. 182

Lehman, D. 466

Levin, S. 318

Levy, M.L. 193

Lew, S.M. 392

Linhares, M.N. 385

Liu, R. 259

Luerssen, T.G. 141

MacDonald, J.D. 308

MacGregor, T.L. 227

Mahadevan, A. 238 
Mahapatra, A.K. 242, 267, 368,472

Maher, C.O. 172, 326

Mahore, A. 39

Maiti, T.K. 238

Malik, N.K. 25

Marcus, R.B., Jr. 227

Martínez-Lage, J.F. 442

Matsota, P. 62

Matuoka, T. 313

Matushita, H. 249

Matzinger, M.A. 283

McMullan, P.J. 462

Meltzer, H. 193

Menascu, S. 82

Mensah Sarfo-Poku, C. 381

Mikrogianakis, A. 283

Mitsuyama, T. 12

Moftakhar, P. 373

Mohapatra, A.K. 245

Moon, K.-S. 205

Moschovi, M. 188, 480

Mottolese, C. 435

Mukherjee, K.K. 46

Muraszko, K.M. 172

Mutiso, B.K. 1

Muzumdar, D. 39, 232

Mwachaka, P.M. 1

Mwangombe, N. 1

Naik, V. 368

Nakano, N. 313

Nanda, M. 138

Nassar, M. 427

Nejat, F. 290

Nestler, U. 34, 294

Neubauer, B.A. 294

Newman, C.B. 193

Nilsson, D. 221
Nistico, L. 127

Nizami, F.A. 25

Nuhoglu, A. 199

Obonyo, N.G. 1

Ohgi, S. 313

Ojemann, J.G. 120

Okada, Y. 12

Osburn, L.L. 71

Panagopoulos, D. 480

Park, D.H. 89

Park, S.H. 299

Parmar, H.A. 326

Patereli, A. 188

Pendleton, C. 475

Per, H. 133

Petrella, J. 155

Pinto, F.C. 249

Pinto, N.C. 249

Post, J.C. 127

Postlethwait, R.A. 329

Preuss, M. 34, 294

Prodromou, N. 188, 480

Qayoom, A. 25

Quigley, M. 127

Quiñones-Hinojosa, A. 475

Ragel, B.T. 390

Ramzan, A.U. 25, 406

Ranger, A. 318

Ranketi, S. 1

Rao, K.L.N. 138

Raskin, J. 390

Rastogi, M. 51

Rathore, Y.S. 472

Redhu, R. 39

Reinstrup, P. 101
Rizvi, S.A.A. 335

Robertson, P.L. 326

Robinson, F.R. 120

Romner, B. 101

Rozzelle, C.J. 19

Rumana, M. 406

Rumeny, P.G. 82

Sack, J.A. 172

Salunke, P. 46

Salvadori, M. 318

Sampath, S. 238

Sarat Chandra, P. 245

Sawada, Y. 313

Sayama, C.M. 308

Schwartz, T.H. 151

Scott, R.M. 373

Seiler, F.A. 392

Senturk, S. 54

Sepehrnia, A. 357

Sfakianos, G. 480

Sgouros, S. 62, 402

Shankar, S.K. 238

Sharma, B.S. 245, 324

Shayanfar, N. 357

Shukla, D. 247

Singh, D.K. 51

Singh, P. 46

Singla, N. 76

Sinha, S. 462

Smith, E.R. 373

Sood, S. 255, 442

Stea, B.D. 381

Stefanaki, K. 188, 480

Stein, M. 34

Stone, J. 19

Stoodley, P. 127

Suri, V. 368

Suwen, L. 313
Szathmari, A. 435

Szymczak, A. 318

Tao, S. 259

Teixeira, M.J. 249

Tomita, T. 303

Tosyalı, N. 110

Trigylidas, T. 283

Tsangaris, I. 62

Tshechmer, S.M. 82

Tucer, B. 133

Undén, J. 101

Vachhrajani, S. 213

Vamsi, K.Y. 245

Vasishta, R.K. 46

Vassilyadi, M. 283

Wani, A.A. 25

Wani, M.A. 25

Whitehead, W.E. 141

Wong, S.-T. 417

Woo, H.J. 299

Wood, D.L. 329

Wood, M.J. 146, 335, 340

Yi, W. 259

Yilmaz, N. 6, 351

Yoon, S.H. 89

Yoshimura, E.M. 249

Yuh, S.J. 283

Zaheer, S.N. 340

Zaki, H.S. 462

Zargar, J. 25

Zetterstrand, S. 221

Zühlke, C.J. 294

Zumwalt, J. 381 